\title{
Activating NOTCH2 Mutation
}

National Cancer Institute

\section{Source}

National Cancer Institute. Activating NOTCH2 Mutation. NCI Thesaurus. Code C158059.

A change in the nucleotide sequence of the NOTCH2 gene that that results in constitutive activation of both neurogenic locus notch homolog protein 2 and its downstream signaling pathways. 